\title{
Medaka Hepatoma
}

National Cancer Institute

\section{Source}

National Cancer Institute. Medaka Hepatoma. NCI Thesaurus. Code C134571.

Hepatoma that occurs in Oryzias latipes. 